\title{
Least square approximate motion generation synthesis of spherical linkages by using Chebyshev and equal spacing
}

\author{
Rasim Alizade ${ }^{a}$, Fatih Cemal Can ${ }^{\mathrm{b}, *}$, Özgür Kilit ${ }^{\mathrm{c}}$ \\ a Mechanical Engineering Department, Izmir Institute of Technology, Urla 35437, Izmir, Turkey \\ b Mechatronics Engineering Department, Izmir Katip Celebi University, Cigli 35620, Izmir, Turkey \\ c Mechanical Engineering Department, Ege University, Bornova 35100, Izmir, Turkey
}

\section{A R T I C L E I N F O}

\section{Article history:}

Received 12 October 2011

Received in revised form 20 September 2012

Accepted 10 October 2012

Available online 28 November 2012

\section{Keywords:}

Rigid body guidance

Approximate motion synthesis

Spherical dyad

Spherical four-bar mechanism

\begin{abstract}
A B S T R A C T
In this paper, approximate motion synthesis of spherical linkages is presented. Rigid body guidance of a spherical four-bar mechanism is performed by a spherical RR open chain. In the first step, position of a point on rigid body and orientation of a rigid body on a unit sphere are described. Synthesizing function of spherical dyad is derived by means of using unit vectors that describe location of two revolute joints and tip point. Being based on the theory of function approximation and besides the linearization of nonlinear synthesis equations by using superposition method, the design procedure for real solutions of fourth order polynomial equation is developed. In the second step, approximate motion generation synthesis of spherical dyad is presented by using least-square approximation. Chebyshev spacing and equal spacing are used in the determination of poses. In the final step, two numerical examples are given to show how error graph is varied in terms of selected poses. The spherical motion generation synthesis of spherical four-bar mechanism is obtained by the combination of the two real solutions of the synthesis of two spherical dyads.
\end{abstract}

(c) 2012 Elsevier Ltd. All rights reserved.

\section{Introduction}

Generally, planar and spherical mechanisms consist of dyads including two moving links connected by two revolute pairs. The kinematic synthesis problem of mechanism can be easily solved by using the kinematic equations of dyads.

Function generation, path generation and motion generation are three main problems in kinematic synthesis of mechanisms. In order to solve synthesis problems, several mathematical methods, such as algebraic method, complex numbers method, vector method, quaternion and biquaternion methods, matrix and screw methods, and CAD based methods were developed.

Levitskii's equation [1] has been introduced for the function generation synthesis of four-bar linkage in the polynomial form and design parameters were synthesized by using the interpolation, least-square and Chebyshev approximation methods. The function generation synthesis of spherical four-bar mechanisms by using analytical methods of polynomial approximation for given four precision positions was studied by Zimmerman [2]. Several studies on the synthesis of function generating spherical linkages have been given in [3]. Alizade and Kilit [4] gave a new methodology for function generation synthesis of spherical four-bar linkage satisfying a specific constraint. This methodology, which has been called by the authors "MDA (minimum deviation area)", is a way for selection of the precision points on given function such that the deviation area between given and generated functions will be minimum. Alizade and Gezgin [5] proposed a new function generation synthesis method of spherical four-bar mechanism with six independent parameters. Interpolation, Chebyshev and least square approximation were used and differences in error graph were compared.

\footnotetext{
* Correponding author at: Department of Mechatronics Engineering, Faculty of Engineering and Architecture, Izmir Katip Celebi University, Cigli Campus 35620, Izzmir, Turkey. Tel.: +90232 329 3535; fax: +902323860888.

E-mail addresses: alizada_rasim@hotmail.com (R. Alizade), f.c.can@hotmail.com (F.C. Can), ozgurkilit@gmail.com (Ö. Kilit).
} 
In the study of Chu and Sun [6], the path generation of spherical four-bar mechanism was solved based on derived theoretical methods and the numerical atlas database of the harmonic characteristic parameters. Mullineux [7] introduced path generation of spherical four-bar mechanism by creating an atlas of the mechanism.

The kinematic mappings have been used to derive the kinematic constraint equations of the dyad using the components of dual quaternion [8]. The constraint manifold of spherical RR dyad fitting for an arbitrary number of location studied in [9,10]. The branching problem in finite position synthesis of spherical four-bar mechanism was studied by McCarthy et al. [11]. For this purpose, rectification theories such as Filemon's construction and Waldron's 'three circle diagram' was directly extended to spherical 4R linkages. Larochelle et al. [12], on the other hand, presented the method for approximating finite set of spatial locations with spherical orientations for spherical linkage. Additionally in this study, a new technique for approximate motion synthesis of spherical RR dyad and spherical 4R closed chains was presented. A CAD program, named SPHINX, for motion synthesis of spherical linkage was developed by Larochelle et al. [13]. Larochelle [14] introduced approximate motion synthesis for planar RR, spherical RR and spatial CC dyads, which are generally part of the kinematic structures in robotic systems and mechanisms. The formulation of synthesis problem was developed by using dual quaternion. Two initial numerical examples were presented after explaining the general approximate motion synthesis procedure. K.Al-Widyan and Angeles [15] recommended a robust analytical solution for rigid body guidance synthesis of spherical mechanism which is one of the complex problems in kinematics. The motion synthesis of spherical 4R linkages for specified rigid body positions was investigated by Ruth and McCarthy [16]. In the study, spherical 4R linkage is designed by using SPHINXPC CAD program. H. Shirazi [17] presented the computer modeling geometric construction of Burmester curve for rigid body guidance synthesis of $4 \mathrm{R}$ spherical linkage in four specified positions on a unit sphere. The sample numerical problem was carried out by using Maple which was one helpful program to calculate the required poles and other variables. Motion generation of an adjustable spherical four-bar was proposed by W.-T. Lee et al. [18]. In the study, the least-square method was used to solve synthesis problem for not limited prescribed rigid-body positions. Synthesis of two-phase adjustable spherical mechanism was explained along with a numerical example.

Approximate motion synthesis of spherical linkages is presented by using least square method in this research. Objective function of spherical dyad is derived by using location of fixed, moving revolute joints and tip point. The motion of the gripper, attached to the second moving link of the spherical RR dyad, is to be guided through a discreet set of $m$ poses $\left\{D_{i}\right\}_{1}^{m}$, starting from the first pose to the last pose. The problem consists of finding the fixed joint center $\mathrm{A}\left(\theta_{A}, \psi_{\mathrm{A}}\right)$ and spherical link lengths $\left(\alpha_{1}, \alpha_{2}\right)$. A discrete set of poses is determined by using Chebyshev and equal spacing. We perform two numerical examples for two spacing methods in the study. These examples show that if the number of poses is increased, maximum and minimum errors will be equal and error will be continuous in the range of poses. In the final part of the study, the spherical four-bar mechanism is designed for seven discrete poses which is not spaced by two spacing methods. The spherical mechanism is constructed with 3 mm sheet metal links, M7 bolts and nuts. This practical study verifies our synthesis methods.

\section{Spherical displacement of a rigid body}

Spherical displacement of a rigid body can be described by three successive angles. These angles are well known as Euler Angles represented by "Leonhard Euler". In this study, ZYX rotation sequence is applied to spherical displacement. In order to illustrate spherical displacement of a rigid body, a reference coordinate system (fixed frame), Oxyz, is established in the center of a unit sphere (see Fig. 1). Spherical displacement of the rigid body on the spherical surface is usually described as

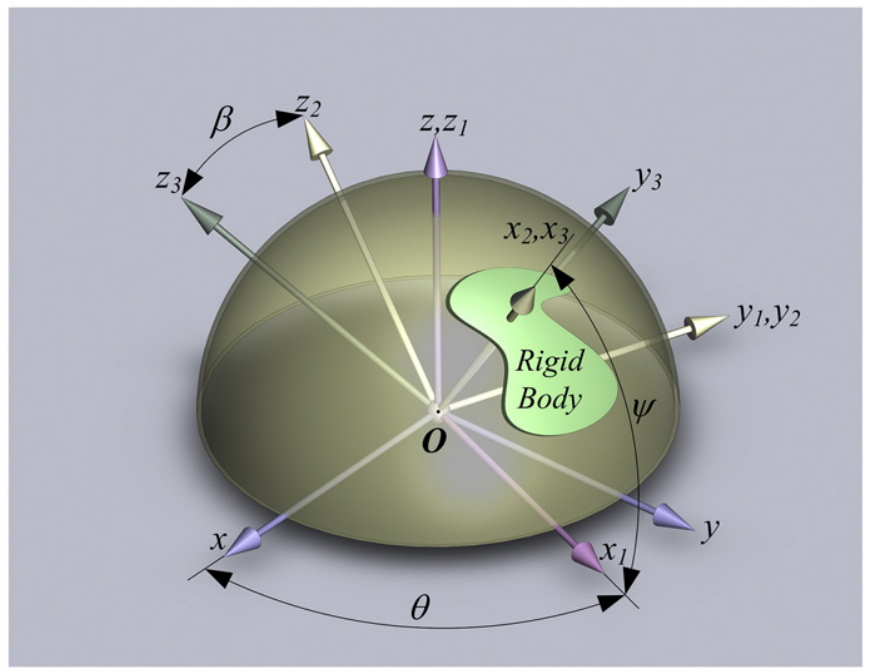

Fig. 1. Spherical displacement of a rigid body. 
position of any point on the rigid body and the orientation about the normal axis of the rigid body. Spherical displacement can be introduced as,

$$
[D]=[\rho][\beta]
$$

where $[\rho]$ defines position of any point on rigid body and $[\beta]$ defines orientation of the rigid body.

As shown in Fig. 1, position of a point on rigid body can be described as two successive rotations firstly around $z$ axis by an angle $\theta$ and then around new axis $y_{1}$ by an angle $\psi$

$$
[\rho]=R_{z}(\theta) R_{y 1}(\psi)=\left[\begin{array}{ccc}
c \theta c \psi & -s \theta & c \theta s \psi \\
s \theta c \psi & c \theta & s \theta s \psi \\
-s \psi & 0 & c \psi
\end{array}\right]
$$

where $c x$ and $s x$ are a shorthand notation for $\cos (x)$ and $\sin (x)$ respectively.

Orientation of the rigid body is represented as a rotation around $x_{2}$ axis by an angle $\beta$ (Fig. 1 ). Orientation of rigid body on sphere is described in matrix form as follows,

$$
[\beta]=R_{X_{2}}(\beta)=\left[\begin{array}{ccc}
1 & 0 & 0 \\
0 & c \beta & s \beta \\
0 & -s \beta & c \beta
\end{array}\right] .
$$

Finally, using Eqs. (1)-(3), the overall spherical displacement matrix of a rigid body on the spherical surface will be:

$$
[D]=R_{z}(\theta) R_{y_{1}}(\psi) R_{x_{2}}(\beta)=\left[\begin{array}{ccc}
c \theta c \psi & -s \theta c \beta+s \beta c \theta s \psi & s \theta s \beta+c \beta c \theta c \psi \\
s \theta c \psi & c \theta c \beta+s \beta s \theta s \psi & -c \theta s \beta+c \beta s \theta s \psi \\
-s \psi & c \psi s \beta & c \beta c \psi
\end{array}\right]
$$

As shown in Eq. (4), a spherical displacement is defined by three independent parameters $(\theta, \psi$ and $\beta)$. Calculated matrices in this section will help us to derive some equations in the next section.

\section{Derivation of objective function for RR spherical dyad}

This section explains how objective function is derived for RR spherical dyad. One RR spherical dyad is depicted in Fig. 2. As shown in Fig. 2, $x_{A}$ and $x_{B}$ represent location of the first (fixed) and the second (moving) revolute joints where as $x_{C}$ describe location of the end effector or the tip point on unit sphere.

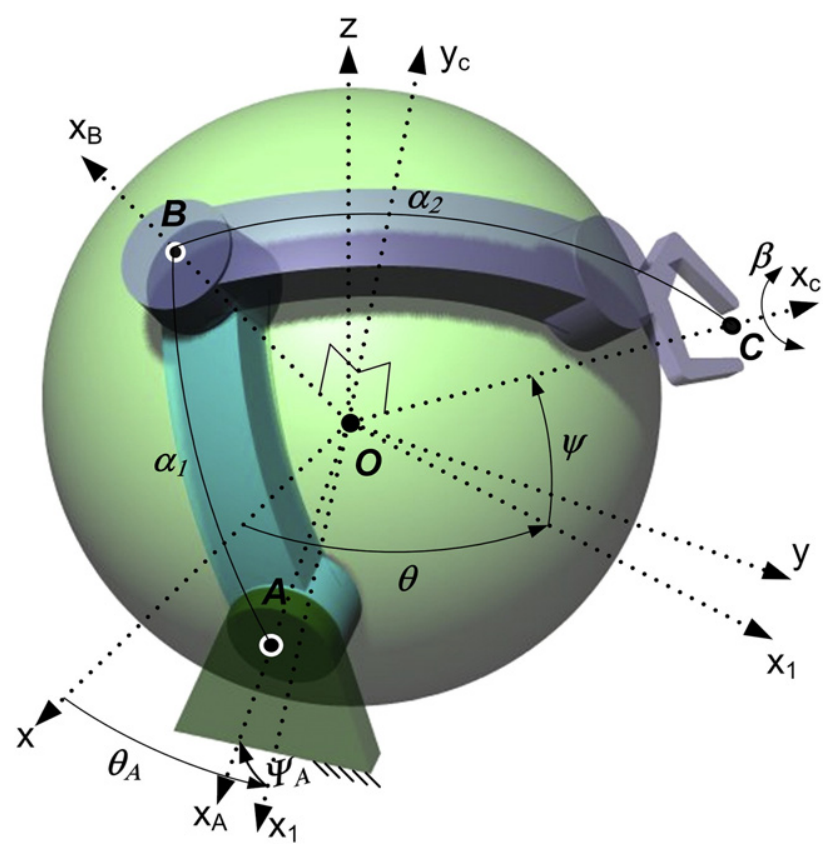

Fig. 2. Spherical RR dyad. 
The position of the fixed revolute joint on the open RR chain is defined by $\theta_{A}$ and $\psi_{A}$. Therefore, the direction of $x_{A}$ can be determined by using the first column of Eq. (2), $x_{A}=\left[\begin{array}{llll}c \theta_{A} c \psi_{A} & s \theta_{A} c \psi_{A} & -s \psi_{A}\end{array}\right]^{T}$. Similarly, the direction of $x_{C}$ is represented by using Eq. (2), $x_{C}=\left[\begin{array}{lll}c \theta c \psi & s \theta c \psi & -s \psi\end{array}\right]^{T}$. The direction of $x_{B}$ is completely unknown and it varies while the first revolute joint is moving, $x_{B}=\left[\begin{array}{lll}u & v & w\end{array}\right]^{T}$.

The axis $y_{C}$ is perpendicular to both axes $x_{B}$ and $x_{C}$. The direction of $y_{C}$ is known from the second column of spherical displacement matrix represented in Eq. (4) $y_{C}=\left[\begin{array}{lll}-s \theta c \beta+s \beta c \theta s \psi & c \theta c \beta+s \beta s \theta s \psi & c \psi s \beta\end{array}\right]^{T}$.

The dyad dimensions $\left\{\alpha_{i}\right\}_{1}^{2}$ are two constant link parameters. The first one is measured between fixed $\left(x_{A}\right)$ and moving $\left(x_{B}\right)$ revolute joints. The second one is measured between moving $\left(x_{B}\right)$ revolute joint and end effector $\left(x_{C}\right)$. These yield two equations as follows;

$$
\begin{aligned}
& \left(x_{A}\right)^{T} \cdot\left(x_{B}\right)=c \alpha_{1} \\
& \left(x_{B}\right)^{T} \cdot\left(x_{C}\right)=c \alpha_{2} .
\end{aligned}
$$

As mentioned before, the axis $y_{C}$ is perpendicular to both axes $x_{B}$ and $x_{C}$. Therefore, we can write:

$$
x_{B} \times x_{C}=y_{C} s \alpha_{2}
$$

The Eqs. (5), (6) and (7) must be opened to develop objective function. Therefore, five scalar equations are by means of dot product and vector product rule. The equations are calculated as follows;

$$
\begin{aligned}
& c \theta_{A} c \psi_{A} u+s \theta_{A} c \psi_{A} v-s \psi_{A} w=c \alpha_{1} \\
& c \theta c \psi u+s \theta c \psi v-s \psi w=c \alpha_{2} \\
& s \psi v+c \psi s \theta w=s \alpha_{2}(s \theta c \beta-s \beta c \theta s \psi) \\
& s \psi u+c \psi c \theta w=s \alpha_{2}(c \theta c \beta+s \beta s \theta s \psi) \\
& s \theta u-c \theta v=s \alpha_{2} s \beta .
\end{aligned}
$$

The elements $(u, v$ and $w)$ of axis $x_{B}$ can be calculated by selecting any three scalar equations from Eqs. (8), (9), (10), (11) and (12) (Eqs. (10), (11) and (12) must not be selected). The Eqs. (9), (10) and (11) are selected to calculate the elements ( $u, v$ and $w$ ) and they are represented in matrix form as follows;

$$
\left[\begin{array}{ccc}
c \theta c \psi & s \theta c \psi & -s \psi \\
0 & s \psi & c \psi s \theta \\
s \psi & 0 & c \psi c \theta
\end{array}\right]\left[\begin{array}{c}
u \\
v \\
w
\end{array}\right]=\left[\begin{array}{c}
c \alpha_{2} \\
s \alpha_{2}(s \theta c \beta-s \beta c \theta s \psi) \\
s \alpha_{2}(c \theta c \beta+s \beta s \theta s \psi)
\end{array}\right]
$$

Unknowns in matrix Eq. (13) are solved and they are found as follows;

$$
\begin{aligned}
& u=c \alpha_{2} c \theta c \psi+s \alpha_{2}(s \theta s \beta+c \theta c \beta s \psi) \\
& v=c \alpha_{2} s \theta c \psi+s \alpha_{2}(s \theta c \beta s \psi-c \theta s \beta) \\
& w=-c \alpha_{2} s \psi+s \alpha_{2} c \beta c \psi
\end{aligned}
$$

The objective function will be obtained by substituting the calculated elements $(u, v$ and $w$ ) into Eq. (8). Note that if these elements are substituted into remaining Eq. (12), it is observed that the right side of the equation is equal to the left side of the equation. After substituting $(u, v$ and $w$ ) into Eq. (8), the objective function will be as follows,

$$
\left(\begin{array}{c}
-c \alpha_{1}\left(s \alpha_{2} c \theta_{A} c \psi_{A}\right)^{-1}+\tan \theta_{A}(-s \beta c \theta+s \theta c \beta s \psi) \\
+\cot \alpha_{2} c \theta c \psi-\tan \psi_{A}\left(c \theta_{A}\right)^{-1} c \beta c \psi+\cot \alpha_{2} \tan \theta_{A} s \theta c \psi \\
+\cot \alpha_{2} \tan \psi_{A}\left(c \theta_{A}\right)^{-1} s \psi+s \beta s \theta+c \theta c \beta s \psi
\end{array}\right)=0
$$

Solution of nonlinear objective function for some given poses is possible by means of various mathematical optimization methods. However, our aim is to develop systematic method for solution of objective function. Our approach is to convert nonlinear terms to linear terms. The spherical RR dyad consist of four constant design parameters $\left\{\alpha_{1}, \alpha_{2}, \theta_{A}, \psi_{A}\right\}$ and three given motion parameters of the gripper $\{\theta, \psi, \beta\}$. The Eq. (17) can be introduced as a difference between polynomial function $\mathrm{P}(C, x)$ and the given continuous function $\mathrm{F}(x)$ :

$$
\Delta(x)=\mathrm{P}(C, x)-\mathrm{F}(x)
$$


where $\mathrm{P}(C, x)$ function can be represented by a polynomial function $\mathrm{P}(C, x)=\sum_{k=1}^{6} p_{k} f_{k}(x)$. Therefore, Eq. (17) will be as follows;

$$
\Delta_{i}=\sum_{k=1}^{6} p_{k} f_{k i}-F_{i}
$$

where $i$ is number of poses, $\left\{p_{k}\right\}$ are constant coefficients, $\left\{f_{k i}\right\}$ are linearly independent continuous functions of motion variables: $p_{1}=c \alpha_{1}\left(s \alpha_{2} c \theta_{A} c \psi_{A}\right)^{-1}, p_{2}=\tan \theta_{A}, p_{3}=\cot \alpha_{2}, p_{4}=\tan \psi_{A}\left(c \theta_{A}\right)^{-1}, p_{5}=\cot \alpha_{2} \tan \theta_{A}, p_{6}=\cot \alpha_{2} \tan \psi_{A}\left(c \theta_{A}\right)^{-1}$, $f_{1 i}=-1, f_{2 i}=-s \beta_{i} c \theta_{i}+s \theta_{i} c \beta_{i} s \psi_{i}, f_{3 i}=c \theta_{i} c \psi_{i}, f_{4 i}=-c \beta_{i} c \psi_{i}, f_{5 i}=s \theta_{i} c \psi_{i}, f_{6 i}=s \psi_{i}$, and $F_{i}=-\left(s \beta_{i} s \theta_{i}+c \theta_{i} c \beta_{i} s \psi_{i}\right)$.

Six coefficients, $\left\{p_{k}\right\}_{1}^{6}$, contain four unknown parameters. It means that only four coefficients are independent while the other two coefficients are the combinations of the others. Looking carefully at $\left\{p_{k}\right\}$ the relations, we can see that $p_{5}$ and $p_{6}$ dependent to the others. This leads us to two additional equations as,

$$
p_{5}=p_{2} p_{3}, \quad p_{6}=p_{4} p_{3}
$$

The motion of the gripper, attached to the second moving link of the spherical RR dyad shown in Fig. 2, is to be guided through a discreet set of $m$ poses $\left\{D_{i}\right\}_{1}^{m}$, starting from reference pose, labeled 1 . The problem consists of finding the fixed joint center $A$ $\left(\theta_{A}, \psi_{A}\right)$ and spherical link lengths $\left(\alpha_{1}, \alpha_{2}\right)$ by using the nonlinear Eqs. (19) and (20).

\section{Solution of nonlinear motion polynomial by linear combination}

In this section, exact solution of motion generation RR dyad is discussed for four poses. As shown in the previous section, there are six unknowns but only four linear equations and two additional nonlinear equations. If $\lambda_{1}=p_{5}$ and $\lambda_{2}=p_{6}$ are defined as non-linear parameters, and if they are taken as known parameters then the approximation function $\mathrm{P}(C, x)=\sum_{k=1}^{6} p_{k} f_{k i}$ will be synthesizing polynomial, consisting of the following four linear terms:

$$
\sum_{k=1}^{4} p_{k} f_{k i}=F_{i}-\lambda_{1} f_{5 i}-\lambda_{2} f_{6 i}
$$

where $i=1, \ldots, 4$. The terms of the left side of the Eq. (21) are linear and constant parameters $p_{k}$ are linearly proportional with non-linear parameters $\lambda_{1} Z$ and $\lambda_{2}$ as follows:

$$
p_{k}=l_{k}+m_{k} \lambda_{i}+n_{k} \lambda_{2} \quad k=1, \ldots, 4
$$

Substituting Eqs. (22) into (21) and separating the real and non-linear coefficient parts, we obtain the following system of linear equations with respect to parameters $l_{k}, m_{k}$, and $n_{k}$ :

$$
\sum_{k=1}^{4} l_{k} f_{k i}=F_{i}, \quad \sum_{K=1}^{4} m_{k} f_{k i}=-f_{5 i}, \quad \sum_{K=1}^{4} n_{k} f_{k i}=-f_{6 i}
$$

The linear coefficients $\left\{l_{k}, m_{k}, n_{k}\right\}_{1}^{4}$ are computed from the system of linear Eq. (23) for the given magnitudes of precision positions and orientations $\left\{\theta_{i}, \psi_{i}, \beta_{i}\right\}_{1}^{4}$ :

$$
\begin{gathered}
{\left[l_{k}\right]_{4 \times 1}=\left[f_{k i}\right]_{4 \times 4}^{-1}\left[F_{i}\right]_{4 \times 1}} \\
{\left[m_{k}\right]_{4 \times 1}=\left[f_{k i}\right]_{4 \times 4}^{-1}\left[-f_{5 i}\right]_{4 \times 1}} \\
{\left[n_{k}\right]_{4 \times 1}=\left[f_{k i}\right]_{4 \times 4}^{-1}\left[-f_{6 i}\right]_{4 \times 1}}
\end{gathered}
$$

The known coefficients $f_{k i}, f_{5 i}, f_{6 i}$ and $F_{i}$ are calculated by using Eq. (19). Solution of linear system of equations is possible, if and only if the determinant of linear system Eq. (24) is non-singular:

$$
\left|f_{k i}\right| \neq 0, \quad k, i=1, \ldots, 4
$$

Solving linear system of Eq. (24), $\left\{l_{k}, m_{k}, n_{k}\right\}_{1}^{4}$ are calculated. Substituting Eq. (22) into Eq. (20) gives the following two non-linear equations:

$$
\begin{aligned}
& \left(l_{2}+m_{2} \lambda_{1}+n_{2} \lambda_{2}\right)\left(l_{3}+m_{3} \lambda_{1}+n_{3} \lambda_{2}\right)=\lambda_{1} \\
& \left(l_{3}+m_{3} \lambda_{1}+n_{3} \lambda_{2}\right)\left(l_{4}+m_{4} \lambda_{1}+n_{4} \lambda_{2}\right)=\lambda_{2} .
\end{aligned}
$$


Firstly, by multiplying Eq. (26) with $n_{4}$ and Eq. (27) with $-n_{2}$, then by summing and arranging, we derive the first equation. Secondly, by dividing Eqs. (26) to (27) and arranging, we obtain second equation. Thus, the two Eqs. (26) and (27) are transformed to the following form:

$$
\begin{aligned}
& a_{1} \lambda_{1}^{2}+a_{2} \lambda_{1} \lambda_{2}+a_{3} \lambda_{1}+a_{4} \lambda_{2}+a_{5}=0 \\
& b_{1} \lambda_{1}^{2}+b_{2} \lambda_{2}^{2}+b_{3} \lambda_{1} \lambda_{2}+b_{4} \lambda_{1}+b_{5} \lambda_{2}=0
\end{aligned}
$$

where $a_{1}=\left(n_{4} m_{2}-m_{4} n_{2}\right) m_{3}, a_{2}=\left(n_{4} m_{2}-m_{4} n_{2}\right) n_{3}, a_{3}=\left(n_{4} l_{2}-l_{4} n_{2}\right) m_{3}+\left(n_{4} m_{2}-m_{4} n_{2}\right) l_{3}-n_{4}, a_{4}=\left(n_{4} l_{2}-l_{4} n_{2}\right) n_{3}+n_{2}, a_{5}=$ $\left(n_{4} l_{2}-l_{4} n_{2}\right) l_{3}, b_{1}=m_{4}, b_{2}=-n_{2}, b_{3}=n_{4}-m_{2}, b_{4}=l_{4}$, and $b_{5}=-l_{2}$.

The non-linear parameter $\lambda_{2}$ can be calculated from Eq. (28) as,

$$
\lambda_{2}=-\frac{a_{1} \lambda_{1}^{2}+a_{3} \lambda_{1}+a_{5}}{a_{2} \lambda_{1}+a_{4}} .
$$

Substituting $\lambda_{2}$ and $\lambda_{2}^{2}$ into Eq. (29) gives a fourth order equation of $\lambda_{1}$ :

$$
c_{4} \lambda_{1}^{4}+c_{3} \lambda_{1}^{3}+c_{2} \lambda_{1}^{2}+c_{1} \lambda_{1}+c 0=0
$$

where $\quad c_{0}=\left(b_{2} a_{5}-a_{4} b_{5}\right) a_{5}, \quad c_{1}=2 b_{2} a_{3} a_{5}-b_{3} a_{4} a_{5}+b_{4} a_{4}^{2}-b_{5} a_{5} a_{2}-b_{5} a_{3} a_{4}, \quad c_{2}=b_{1} a_{4}^{2}+b_{2} a_{3}^{2}+2 b_{2} a_{1} a_{5}-b_{3} a_{4} a_{3}+2 b_{4} a_{2} a_{4}-$ $b_{5} a_{3} a_{2}-b_{5} a_{1} a_{4}-b_{3} a_{2} a_{5}, c_{3}=2 b_{1} a_{2} a_{4}+2 b_{2} a_{1} a_{3}-b_{3} a_{2} a_{3}-b_{3} a_{4} a_{1}+b_{4} a_{2}^{2}-b_{5} a_{1} a_{2}$, and $c_{4}=b_{1} a_{2}^{2}+b_{1} a_{1}^{2}-b_{3} a_{2} a_{1}$.

Mathematica software package is used to obtain solution of the fourth order algebraic Eq. (31) that gives values of the first nonlinear coefficient $\lambda_{1}$. The second nonlinear coefficient $\lambda_{2}$ is numerically calculated by substituting these $\lambda_{1}$ values into Eq. (30). Nonlinear coefficients $\lambda_{1}$ and $\lambda_{2}$ are then used to find the constant coefficients $\left\{p_{k}\right\}_{1}^{4}$ in Eq. (22). Finally, using these values, the four design parameters $\left\{a_{1}, a_{2}, \theta_{A}, \psi_{A}\right\}$ of spherical dyad can be found from Eq. (19) as

$$
\theta_{A}=\tan ^{-1} p_{2}, \quad \psi_{A}=\tan ^{-1}\left(p_{4} c \theta_{A}\right), \quad \alpha_{2}=\cot ^{-1} p_{3}, \quad a_{1}=\cos ^{-1}\left(p_{1} s \alpha_{2} c \theta_{A} c \psi_{A}\right) .
$$

Exact solution for motion generation synthesis is just completed. Generally, four poses is very limited to design spherical mechanisms. The more poses is needed to design such mechanisms. Therefore, solution of synthesis problem can be achieved by using optimization methods such least-square approximation. This section is the base for least-square approximation in the next section.

\section{Motion generation synthesis of spherical dyad by least-square approximation}

In the least-square approximation for the range of angular positions $\left\{\theta_{i}, \psi_{i}\right\}_{1}^{m}$ and precision orientations $\left\{\beta_{i}\right\}_{1}^{m}$, the unknown parameters are computed by taking the minimum of the squares of the errors between generated $p_{k} f_{k i}$ function and given $F_{i}$ function.

$$
\Delta^{2}=\frac{S}{m-1} \quad \text { where } S=\sum_{i=1}^{m}\left[\sum_{k=1}^{4} p_{k} f_{k i}-F_{i}\right]^{2}
$$

where $m$ index is the number of given data which is greater than the number of unknowns. The linear coefficients $l_{k}, m_{k}$ and $n_{k}$ that were described by Eq. (24) can also be calculated similarly to Eq. (33) from the following equations:

$$
\left.\begin{array}{c}
S_{1}=\sum_{i=1}^{m}\left[\sum_{k=1}^{4} l_{k} f_{k i}-F_{i}\right]^{2}, S_{2}=\sum_{i=1}^{m}\left[\sum_{k=1}^{4} m_{k} f_{k i}+f_{5 i}\right]^{2}, \\
S_{3}=\sum_{i=1}^{m}\left[\sum_{k=1}^{4} n_{k} f_{k i}+f_{6 i}\right]^{2}
\end{array}\right\} .
$$

Since the number of equations exceeds the number of unknowns in Eq. (34), i.e. $m>4$, synthesis problem leads to an over determined system. But the optimum solution can be found in the least-square sense if the partial derivation from $\left\{S_{j}\right\}_{1}^{3}$ equals to zero respect to unknown coefficients:

$$
\frac{\partial S_{1}}{\partial l_{k}}=0, \quad \frac{\partial S_{2}}{\partial m_{k}}=0, \quad \frac{\partial S_{3}}{\partial n_{k}}=0 .
$$

Applying the conditions in Eq. (35) to the system of linear Eq. (34) yields:

$$
\left[c_{k l}\right]\left[l_{k}\right]=\left[F_{k}\right], \quad\left[c_{k l}\right]\left[m_{k}\right]=\left[f_{5 k}\right], \quad\left[c_{k l}\right]\left[n_{k}\right]=\left[f_{6 k}\right]
$$

where $c_{k l}=c_{l k}=\sum_{i=1}^{m} f_{k i} f_{l i}, \quad F_{k}=\sum_{i=1}^{m} F_{i} f_{k i}, \quad f_{5 k}=-\sum_{i=1}^{m} f_{5 i} f_{k i}$ and $f_{6 k}=-\sum_{i=1}^{m} f_{6 i} f_{k i}, k=1 \ldots 4, l=1 \ldots 4$. 
Table 1

Nine poses for spherical dyad (Chebyshev spacing).

\begin{tabular}{lll}
\hline$i$ & $\theta_{i}\left({ }^{\circ}\right)$ & $\psi_{i}\left({ }^{\circ}\right)$ \\
\hline 1 & 300.38 & -9.97721 \\
2 & 303.349 & -9.79904 \\
3 & 308.93 & 0.0759612 \\
4 & 316.449 & -9.46418 \\
5 & 325 & -9.01303 \\
6 & 333.551 & -8.5 \\
7 & 341.07 & -7.98697 \\
8 & 346.651 & -7.53582 \\
9 & 349.62 & -7.20096 \\
\hline
\end{tabular}

The known linear independent functions $\left\{f_{k i}\right\}_{1}^{m}$, and also the given functions: $\left\{F_{i}\right\}_{1}^{m},\left\{f_{5 i}\right\}_{1}^{m},\left\{f_{6 i}\right\}_{1}^{m}$ for $m>4$ poses of a rigid body on sphere are computed by using Eq. (19). The determinant of linear Eq. (36) has to be non-zero in a similar fashion that in Eq. (25),

$$
\left|c_{k l}\right| \neq 0
$$

Solving set of linear Eq. (36) yields a solution for linear parameters $\left\{l_{k}, m_{k}, n_{k}\right\}_{1}^{4}$ with respect to $m>4$ poses of rigid body on sphere as follows:

$$
\left[l_{k}\right]=\left[c_{k l}\right]^{-1}\left[F_{k}\right], \quad\left[m_{k}\right]=\left[c_{k l}\right]^{-1}\left[f_{5 k}\right], \quad\left[n_{k}\right]=\left[c_{k l}\right]^{-1}\left[f_{6 k}\right]
$$

Using the results of Eq. (38), the values of coefficients $\left\{c_{j}\right\}_{0}^{4}$ of fourth order equation are defined by using Eqs. (28), (29) and (31). Solving Eqs. (31) and (30) gives the $\lambda_{1}$ and $\lambda_{2}$ nonlinear parameters. Finally, the constant coefficients of polynomial functions are computed by Eq. (22) and four design parameters $\left\{\alpha_{1}, \alpha_{2}, \theta_{A}, \psi_{A}\right\}$ are calculated with respect to Eq. (32). Usually, least-square approximation gives small error between precision poses of approximation but in some sub ranges an error might be greater than the middle values. The error of objective function depends on given poses. Selection of random poses end up with random errors. However, if poses are calculated by a function or an equation (Chebyshev spacing is used in this study), the error can be controlled between start and final pose. Moreover, maximum and minimum errors can be equal.

\section{Chebyshev spacing}

Chebyshev spacing is a well-known method to define precision points. This method was usually used for function generation synthesis. However, in this study, same approach is used to determine poses for motion generation of a spherical dyad. For $m$ poses in the range $\theta_{0} \leq \theta \leq \theta_{m+1}, \psi_{0} \leq \psi \leq \psi_{m+1}$ and $\beta_{0} \leq \beta \leq \beta_{m+1}$, the poses $\theta_{i}, \psi_{i}$ and $\beta_{i}$, according to Chebyshev spacing are given by;

$$
\begin{gathered}
\theta_{i}=\frac{\theta_{m+1}+\theta_{0}}{2}-\frac{\theta_{m+1}+\theta_{0}}{2} \cos \frac{\pi(2 i-1)}{2 m}, \\
\psi_{i}=\frac{\psi_{m+1}+\psi_{0}}{2}-\frac{\psi_{m+1}-\psi_{0}}{2} \cos \frac{\pi(2 i-1)}{2 m}, \\
\beta_{i}=\frac{\beta_{m+1}-\beta_{0}}{2}+\frac{\beta_{m+1}-\beta_{0}}{2} \cos \frac{\pi(2 i-1)}{2 m}, \\
i=1,2, \ldots, m .
\end{gathered}
$$

\begin{tabular}{|c|c|c|c|}
\hline$i$ & $\theta_{i}\left({ }^{\circ}\right)$ & $\psi_{i}\left({ }^{\circ}\right)$ & $\beta_{i}\left({ }^{\circ}\right)$ \\
\hline 1 & 300 & -10 & 0 \\
\hline 2 & 306.25 & -9.625 & 1.25 \\
\hline 3 & 312.5 & -9.25 & 2.5 \\
\hline 4 & 318.75 & -8.875 & 3.75 \\
\hline 5 & 325 & -8.5 & 5 \\
\hline 6 & 331.25 & -8.125 & 6.25 \\
\hline 7 & 337.5 & -7.75 & 7.5 \\
\hline 8 & 343.75 & -7.375 & 8.75 \\
\hline 9 & 350 & -7 & 10 \\
\hline
\end{tabular}

For an example, assume that we want to design a dyad to generate a motion for the ranges $300^{\circ} \leq \theta \leq 350^{\circ},-10^{\circ} \leq \psi \leq-7^{\circ}$ and $0^{\circ} \leq \beta \leq 10^{\circ}$ using nine poses $(m=9)$. Then, the nine poses are calculated by using Eq. (39) and they are listed in Table 1.

Table 2

Nine poses for spherical dyad (equal spacing). 
Table 3

Linearly independent continuous functions for equal spacing.

\begin{tabular}{|c|c|c|c|c|c|c|c|}
\hline$i$ & $F_{i}$ & $f_{1 i}$ & $f_{2 i}$ & $f_{3 i}$ & $f_{4 i}$ & $f_{5 i}$ & $f_{6 i}$ \\
\hline 1 & 0.086824 & -1 & 0.150384 & 0.492404 & -0.984808 & -0.852869 & -0.173648 \\
\hline 2 & 0.116435 & -1 & 0.121905 & 0.582986 & -0.985689 & -0.795092 & -0.167199 \\
\hline 3 & 0.140652 & -1 & 0.088930 & 0.666805 & -0.986057 & -0.727690 & -0.160743 \\
\hline 4 & 0.158868 & -1 & 0.052333 & 0.742838 & -0.985912 & -0.651452 & -0.154279 \\
\hline 5 & 0.170608 & -1 & 0.013064 & 0.810154 & -0.985252 & -0.567276 & -0.147809 \\
\hline 6 & 0.175538 & -1 & -0.027871 & 0.867926 & -0.984078 & -0.476161 & -0.141333 \\
\hline 7 & 0.173470 & -1 & -0.069427 & 0.915441 & -0.982389 & -0.379188 & -0.134851 \\
\hline 8 & 0.164369 & -1 & -0.110544 & 0.952108 & -0.980185 & -0.277514 & -0.128363 \\
\hline 9 & 0.148348 & -1 & -0.150169 & 0.977467 & -0.977467 & -0.172354 & -0.121869 \\
\hline
\end{tabular}

\section{Equal spacing}

Equal spacing is to divide the range of poses into equal intervals. For $m$ poses in the range $\theta_{0} \leq \theta \leq \theta_{m+1}, \psi_{0} \leq \psi \leq \psi_{m+1}$ and $\beta_{0} \leq \beta \leq \beta_{m+1}$, the poses $\theta_{i}, \psi_{i}$ and $\beta_{i}$, according to equal spacing are given as follows;

$$
\begin{gathered}
\theta_{i}=\theta_{0}-(i-1) \frac{\theta_{0}-\theta_{m+1}}{(m-1)}, \quad \psi_{i}=\psi_{0}-(i-1) \frac{\psi_{0}-\psi_{m+1}}{(m-1)}, \\
\beta_{i}=\beta_{0}-(i-1) \frac{\beta_{0}-\beta_{m+1}}{(m-1)}, \quad i=1,2, \ldots, m .
\end{gathered}
$$

For an example, assume that we want to design a dyad to generate a motion for the ranges $300^{\circ} \leq \theta \leq 350^{\circ},-10^{\circ} \leq \psi \leq-7^{\circ}$ and $0^{\circ} \leq \beta \leq 10^{\circ}$ using nine poses $(m=9)$. Then, the nine poses are calculated by using Eq. (40) and they are listed in Table 2.

\section{Numerical example for equal spacing}

In this example, a motion generation synthesis of a spherical four-bar linkage is attempted by least square approximation and equal spacing. The spherical four-bar linkage can be constructed by combining two real results of synthesized spherical dyads. The design of spherical dyad is performed by determining nine poses of the rigid body on the sphere. Nine poses, which was calculated by using equal spacing formulations, were given in Table 2. These poses are going to be used in this example. The magnitudes of linearly independent and continuous functions of the motion are calculated from Eq. (19) and listed in Table 3.

Using values of precision poses from Table 2 and linearly independent continuous functions from Table 3 , the matrices in Eqs. (36) and (37) give;

$$
\begin{aligned}
{\left[c_{k l}\right] } & =\left[\begin{array}{llll}
9 & -0.068605 & -7.00813 & 8.85184 \\
-0.068605 & 0.088662 & -0.085906 & -0.069646 \\
-7.00813 & -0.085906 & 5.6884 & -6.889760 \\
8.851840 & -0.069646 & -6.889760 & 8.706180
\end{array}\right], \\
{\left[F_{k}\right] } & =\left[\begin{array}{llll}
-1.33511 & -0.007081 & 1.073310 & -1.312960
\end{array}\right]^{T}, \\
{\left[f_{5 k}\right.} & =\left[\begin{array}{llll}
-4.8996 & -0.235244 & 3.50531 & -4.82393
\end{array}\right]^{T}, \text { and } \\
{\left[f_{6 k}\right.} & =\left[\begin{array}{llll}
-1.33009 & 0.025004 & 1.01197 & -1.30855
\end{array}\right]^{T} .
\end{aligned}
$$

By using system of linear Eq. (38), we calculate linear coefficients $l_{k}, m_{k}, n_{k}$ as;

$$
\begin{aligned}
& {\left[l_{k}\right]=\left[\begin{array}{llll}
9.12115 & -0.087027 & 0.213043 & -9.25665
\end{array}\right]^{T},} \\
& {\left[m_{k}\right]=\left[\begin{array}{llll}
23.474 & 0.484695 & -0.724381 & -24.9902
\end{array}\right]^{T},} \\
& {\left[n_{k}\right]=\left[\begin{array}{llll}
2.30338 & -0.0930379 & -0.125162 & -2.59201
\end{array}\right]^{T} .}
\end{aligned}
$$

Table 4

Nonlinear terms and constant coefficients (equal spacing).

\begin{tabular}{lccccc}
\hline$\lambda_{1}$ & $\lambda_{2}$ & $p_{1}$ & $p_{2}$ & $p_{3}$ & $p_{4}$ \\
\hline-0.177486 & -4.94473 & -6.43477 & 0.286993 & 0.960501 & -1.99556 \\
0.047531 & 2.64996 & 16.3407 & -0.310535 & -0.15306 & -13.3132 \\
0.07212 & -2.29148 & 5.53594 & 0.161124 & 0.447606 & -5.11941 \\
3.11208 & -23.2465 & 28.6287 & 3.58419 & 0.86828 & -26.773 \\
\hline
\end{tabular}


Table 5

Design parameters of spherical dyad (equal spacing).

\begin{tabular}{lcrr}
\hline$\#$ & $\theta_{A}\left({ }^{\circ}\right)$ & $\psi_{A}\left({ }^{\circ}\right)$ & $\alpha_{2}\left({ }^{\circ}\right)$ \\
\hline 1 & -17.2514 & -86.5389 & 158.633 \\
2 & 9.15303 & -78.8083 & 14.4806 \\
3 & 74.4107 & -82.0874 & 65.8864 \\
\hline
\end{tabular}

The coefficients $\left\{c_{l}\right\}_{0}^{4}$ of the fourth order Eq. (31) are computed by using the values of linear coefficients $l_{k}, m_{k}, n_{k}$. The forth order equation is found as follows; $-0.819699 \lambda_{1}^{4}+2.51519 \lambda_{1}^{3}+0.165962 \lambda_{1}^{2}-0.045071 \lambda_{1}+0.001547=0$. Four roots of the equation are calculated and then the second nonlinear is found by means of Eq. (30). Once two nonlinear terms are found, constant coefficients $\left\{p_{k}\right\}_{1}^{4}$ in Eq. (22) are also determined. Four roots of nonlinear terms and corresponding constant coefficients are listed in Table 4.

Although we have four result, the first one is not usable due to the fact that it does not satisfy Eq. (20). Remember that $\lambda_{1}$ must be equal to multiplication of $p_{2}$ and $p_{3}$. Similarly, $\lambda_{2}$ must be equal to multiplication of $p_{3}$ and $p_{4}$. Only the first result does not obey these conditions. Therefore, the first result is removed from our solution set. Three different design parameters are calculated by substituting usable results into Eq. (32). They are tabulated in Table 5.

At first look, we have three design parameters that are usable to construct a spherical dyad. But, all of them are really usable or some of them are not physically realizable. In the first one, design parameter $\alpha_{2}$ has minus sign. This shows that this is not usable design parameters at \#1. Therefore, two different parameters (\#2 and \#3) can be used to construct a spherical dyad (see Fig. 3). If these two dyads are combined, the four-bar spherical mechanism is constructed to generate desired motion.

\section{Numerical example for Chebyshev spacing}

Similar to previous example, a motion generation synthesis of a spherical four-bar linkage is attempted by least square approximation but this time Chebyshev spacing is used. Firstly, nine precision poses of the rigid body on the sphere should be determined to design the spherical dyad. These poses, which were previously calculated by using Chebyshev spacing formulations, were given in Table 1. The magnitudes of linearly independent and continuous functions of the motion are calculated from Eq. (19) and listed in Table 6.

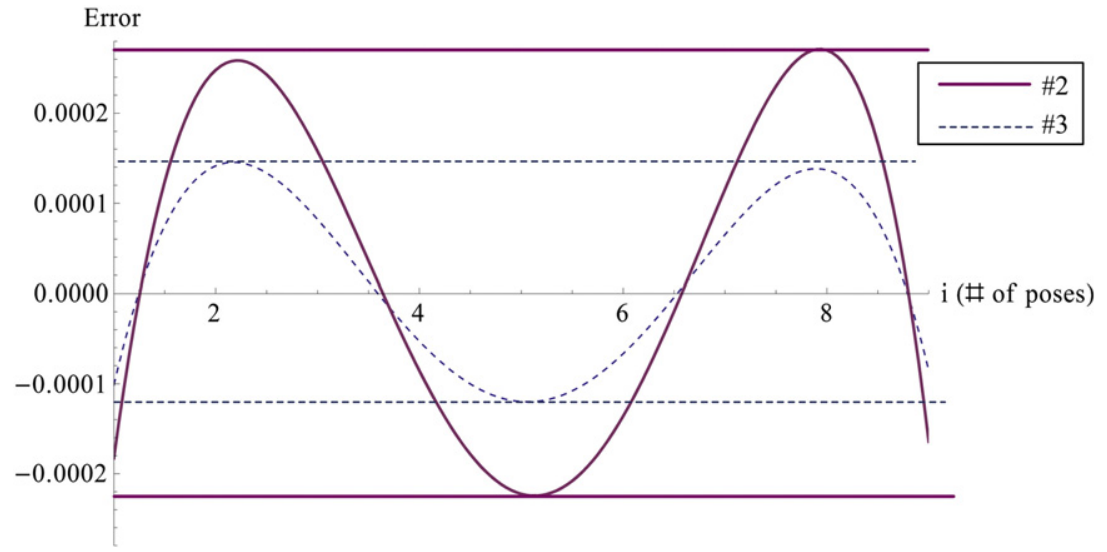

Fig. 3. Errors graph for equal spacing.

Table 6

Linearly independent continuous functions for Chebyshev spacing.

\begin{tabular}{|c|c|c|c|c|c|c|c|}
\hline$i$ & $F_{i}$ & $f_{1 i}$ & $f_{2 i}$ & $f_{3 i}$ & $f_{4 i}$ & $f_{5 i}$ & $f_{6 i}$ \\
\hline 1 & 0.088765 & -1 & 0.148796 & 0.498081 & -0.984876 & -0.849645 & -0.173256 \\
\hline 2 & 0.103322 & -1 & 0.135731 & 0.541722 & -0.985343 & -0.823147 & -0.170193 \\
\hline 3 & 0.127520 & -1 & 0.108266 & 0.619822 & -0.985909 & -0.767322 & -0.164431 \\
\hline 4 & 0.152894 & -1 & 0.066166 & 0.715818 & -0.986025 & -0.680487 & -0.156659 \\
\hline 5 & 0.170608 & -1 & 0.013064 & 0.810154 & -0.985252 & -0.567276 & -0.147809 \\
\hline 6 & 0.175596 & -1 & -0.043151 & 0.886642 & -0.983516 & -0.441088 & -0.138948 \\
\hline 7 & 0.169130 & -1 & -0.093033 & 0.937744 & -0.981193 & -0.321616 & -0.131146 \\
\hline 8 & 0.157782 & -1 & -0.129183 & 0.965306 & -0.978988 & -0.229067 & -0.125350 \\
\hline 9 & 0.149515 & -1 & -0.147823 & 0.976255 & -0.977467 & -0.178821 & -0.122264 \\
\hline
\end{tabular}


Table 7

Nonlinear terms and constant coefficients (Chebyshev spacing).

\begin{tabular}{lccrr}
\hline$\lambda_{1}$ & $\lambda_{2}$ & $p_{1}$ & $p_{2}$ & $p_{3}$ \\
\hline-0.177533 & -4.9483 & -6.44156 & 0.287141 & 0.960916 \\
0.047521 & 2.65015 & 16.3511 & -0.31064 & -0.152979 \\
0.072195 & -2.29391 & 5.53912 & 0.161205 & -17.3236 \\
3.10898 & -23.2336 & 28.6103 & 3.5801 & 0.447845 \\
\hline
\end{tabular}

Using values of precision poses from Table 1 and linearly independent continuous functions from Table 6 , the matrices in Eqs. (36) and (37) give;

$$
\begin{aligned}
& {\left[c_{k l}\right]=\left[\begin{array}{llll}
9 & -0.058833 & -6.95155 & 8.84875 \\
-0.058833 & 0.10589 & -0.121821 & -0.06043 \\
-6.95155 & -0.121821 & 5.64486 & -6.83103 \\
8.84875 & -0.06043 & -6.83103 & 8.70618
\end{array}\right],} \\
& {\left[F_{k}\right]=\left[\begin{array}{llll}
-1.29513 & -0.012413 & 1.03945 & -1.27309
\end{array}\right]^{T},} \\
& {\left[f_{5 k}\right]=\left[\begin{array}{llll}
-4.85847 & 0.268682 & 3.37977 & -4.78274
\end{array}\right]^{T}, \text { and }} \\
& {\left[f_{6 k}\right]=\left[\begin{array}{llll}
-1.33006 & 0.026516 & 0.998839 & -1.30813
\end{array}\right]^{T} .}
\end{aligned}
$$

By using system of linear Eq. (38), we calculate linear coefficients $l_{k}, m_{k}, n_{k}$ as;

$$
\begin{aligned}
& {\left[l_{k}\right]=\left[\begin{array}{llll}
9.12897 & -0.087146 & 0.213084 & -9.26455
\end{array}\right]^{T},} \\
& {\left[m_{k}\right]=\left[\begin{array}{llll}
23.4847 & 0.484425 & -0.724395 & -25.001
\end{array}\right]^{T},} \\
& {\left[n_{k}\right]=\left[\begin{array}{llll}
2.30407 & -0.09302 & -0.12514 & -2.59269
\end{array}\right]^{T} .}
\end{aligned}
$$

The coefficients $\left\{c_{l}\right\}_{0}^{4}$ of the fourth order Eq. (31) are computed by using the values of linear coefficients $l_{k}, m_{k}, n_{k}$. The forth order equation is found as follows; $-0.817872 \lambda_{1}^{4}+2.49546 \lambda_{1}^{3}+0.161589 \lambda_{1}^{2}-0.045817 \lambda_{1}+0.001549=0$. Four roots of the equation are calculated and then the second nonlinear is found by means of Eq. (30). Once two nonlinear terms are found, constant coefficients $\left\{p_{k}\right\}_{1}^{4}$ in Eq. (22) are also determined. Four roots of nonlinear terms and corresponding constant coefficients are listed in Table 7.

The first result must be removed, because it does not satisfy Eq. (20). This equation reminds that $\lambda_{1}$ must be equal to multiplication of $p_{2}$ and $p_{3} . \lambda_{2}$ must be also equal to multiplication of $p_{3}$ and $p_{4}$. The first result does not obey these conditions when others do. Therefore, our solution set is reduced by one result. Three design parameters are calculated by substituting usable results into Eq. (32). They are tabulated in Table 8.

Table 8

Design parameters of spherical dyad (Chebyshev spacing).

\begin{tabular}{lrrr}
\hline$\#$ & $\theta_{A}\left({ }^{\circ}\right)$ & $\psi_{A}\left({ }^{\circ}\right)$ & $\alpha_{1}\left({ }^{\circ}\right)$ \\
\hline 1 & -17.2569 & -86.5409 & 158.639 \\
2 & 9.1576 & -78.8139 & 14.4858 \\
3 & 74.3938 & -82.0902 & 36.8955 \\
\hline
\end{tabular}

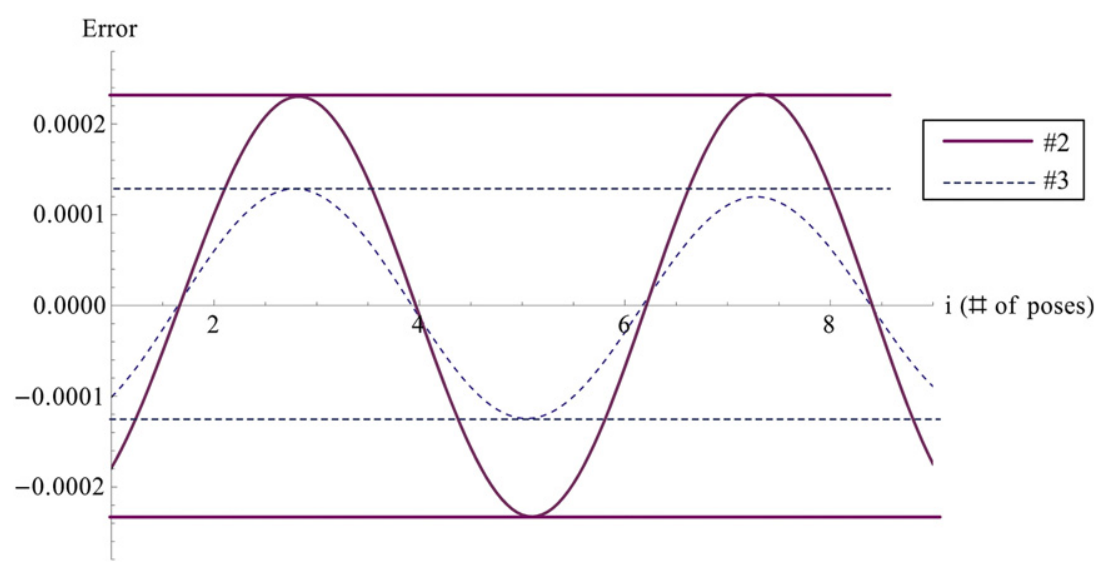

Fig. 4. Errors graph for Chebyshev spacing with nine poses. 


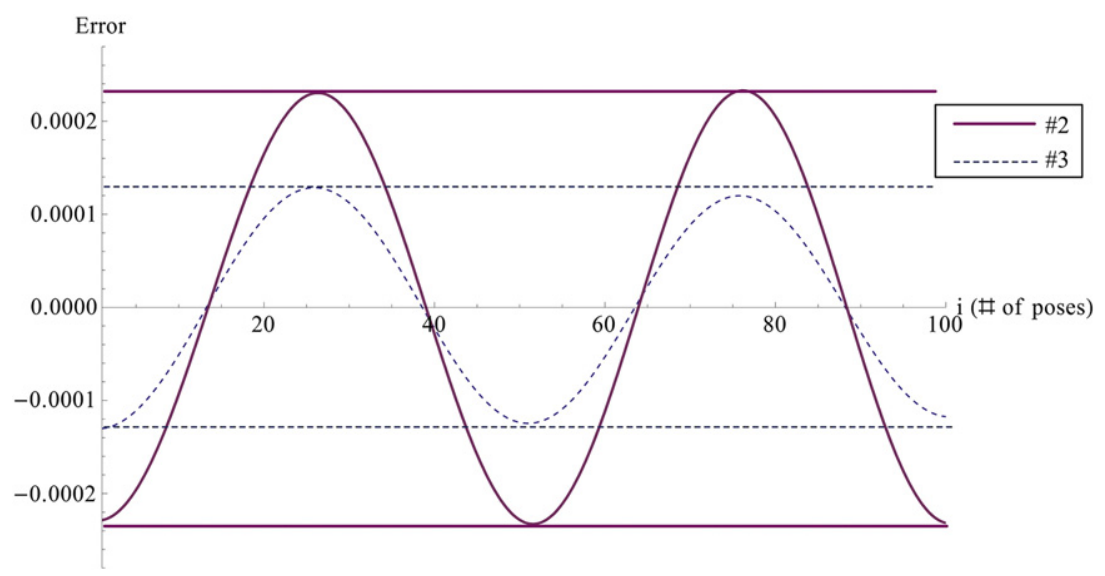

Fig. 5. Errors graph for Chebyshev spacing with one hundred poses.

Firstly, we use nine poses. Errors graph is depicted in Fig. 4. Then, the synthesis is repeated for one hundred poses. If two graphs are compared, maximum and minimum errors will be equal when the poses are increased (Fig. 5). For Chebyshev spacing, the more poses are used, the best design parameters are obtained.

\section{Practical example}

In this section of the study, design and construction of a spherical four-bar mechanism will be explained. This is different from previous examples due to the fact that discrete poses are used for this example. Seven discrete poses are shown in Table 9. These poses are neither equally spaced nor spaced by using Chebyshev formulation.

The linearly independent continuous functions, matrices and other coefficients are calculated by means of a Mathematica notebook. These coefficients and parameters are not important for the construction of the mechanism. Therefore, it is not necessary to give all numerical values. In the final of Mathematica notebook, we calculate the design parameters which are real and physically realizable. The calculated design parameters are given in Table 10.

Four different parts are manufactured in order to construct designed spherical mechanism. The parts are produced from 3 mm sheet steel. They are formed by a laser cutting machine. The produced links are depicted in Table 11 . Number of holes in each link is more than required two holes. The reason is to reshape these links in circular form.

The links can be bonded from the holes and they can be easily reshaped depending on the selected materials ( $3 \mathrm{~mm}$ sheet metal can be bonded by using pliers and other tools). The four links are shaped in circular form. The fourth link is shown in Fig. 6(a) after reshaping. The most important rule is to make the axes of all holes intersect at center point 0 . We control the shape of links by using circular shaped CD-ROM or DVD disk (Fig. 6).

After reshaping all links, spherical four-bar mechanism is constructed by assembling necessary links with bolts and nuts. The spherical four-bar mechanism is assembled according to design parameters which we found from our calculations. The some poses of constructed mechanism are shown in Fig. 7. It is seen that the mechanism moves nearly through desired poses.

Table 9

Seven discrete poses for practical example.

\begin{tabular}{|c|c|c|c|}
\hline$i$ & $\theta_{1}\left({ }^{\circ}\right)$ & $\psi_{1}\left({ }^{\circ}\right)$ & $\beta_{1}\left({ }^{\circ}\right)$ \\
\hline 1 & 120 & -11 & -13 \\
\hline 2 & 108 & -40 & -9 \\
\hline 3 & 91 & -45 & 2 \\
\hline 4 & 75 & -41 & 24 \\
\hline 5 & 60 & -33 & 4 \\
\hline 6 & 58 & -26 & 8 \\
\hline 7 & 55 & -10 & -5 \\
\hline
\end{tabular}

Table 10

Design parameters of spherical dyad.

\begin{tabular}{llll}
\hline$\#$ & $\theta_{A}\left({ }^{\circ}\right)$ & $\psi_{A}\left({ }^{\circ}\right)$ & $\alpha_{2}\left({ }^{\circ}\right)$ \\
\hline 1 & -32.3137 & -66.1943 & 28.809 \\
2 & 89.3205 & -25.9181 & 30.637 \\
\hline
\end{tabular}


Table 11

Produced links for the construction of spherical mechanism.

\#

\section{Conclusion}

This research proposed approximation motion synthesis of a spherical dyad. The objective function is derived in terms of poses and four design parameters. In this function, nonlinear terms are converted to linear terms by using linear combination. The poses which are required for synthesis are determined by two spacing methods such as Chebyshev and equal spacing. All calculations are realized by a prepared Mathematica notebook. Chebyshev spacing shows that the better results will be obtained if the more precision poses are used. Finally, a real spherical four-bar mechanism is constructed to verify our synthesis methods.

In the practical example, two different parameters are obtained when motion of spherical RR dyad is synthesized. Therefore, we have two different RR dyads that generate same spherical motion. Then, their floating links are assembled to form a coupler of closed spherical kinematic chain. Advantages of constructing an $4 \mathrm{R}$ spherical linkage by using these RR dyads:

1. Motion generation problem of R-R open kinematic chains requires eliminating only one joint variable (the first joint variable). However, two joint variables (crank and rocker joint variables) have to be eliminated in the motion generation problem of $4 \mathrm{R}$ spherical linkage. Therefore, problem of motion generation $4 \mathrm{R}$ spherical linkage is simplified by using motion generation problem of R-R open chain. The mathematical model in R-R open synthesis method is simpler than $4 \mathrm{R}$ spherical linkage model.

a

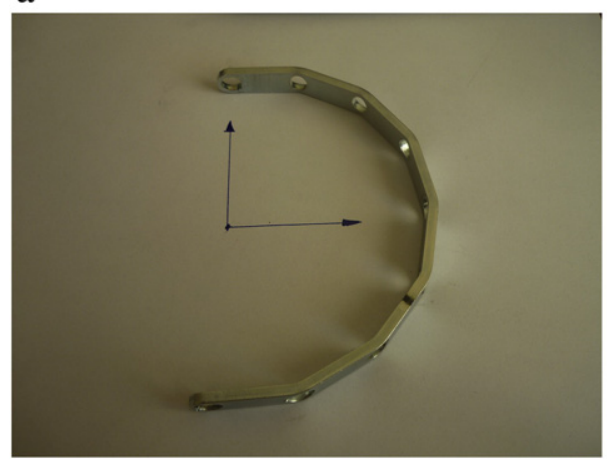

b

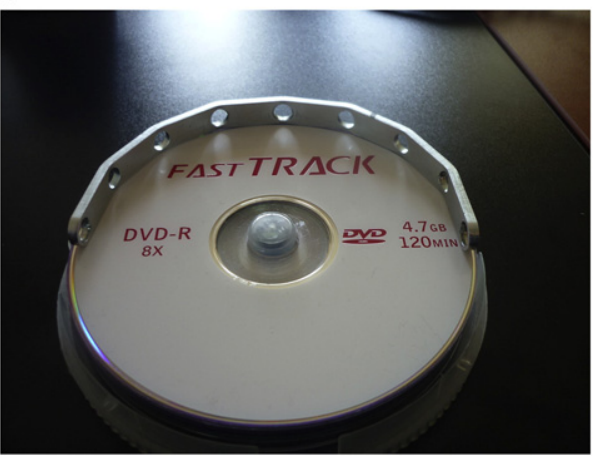

Fig. 6. (a) The fourth link after reshaping and (b) control of the circular shape. 

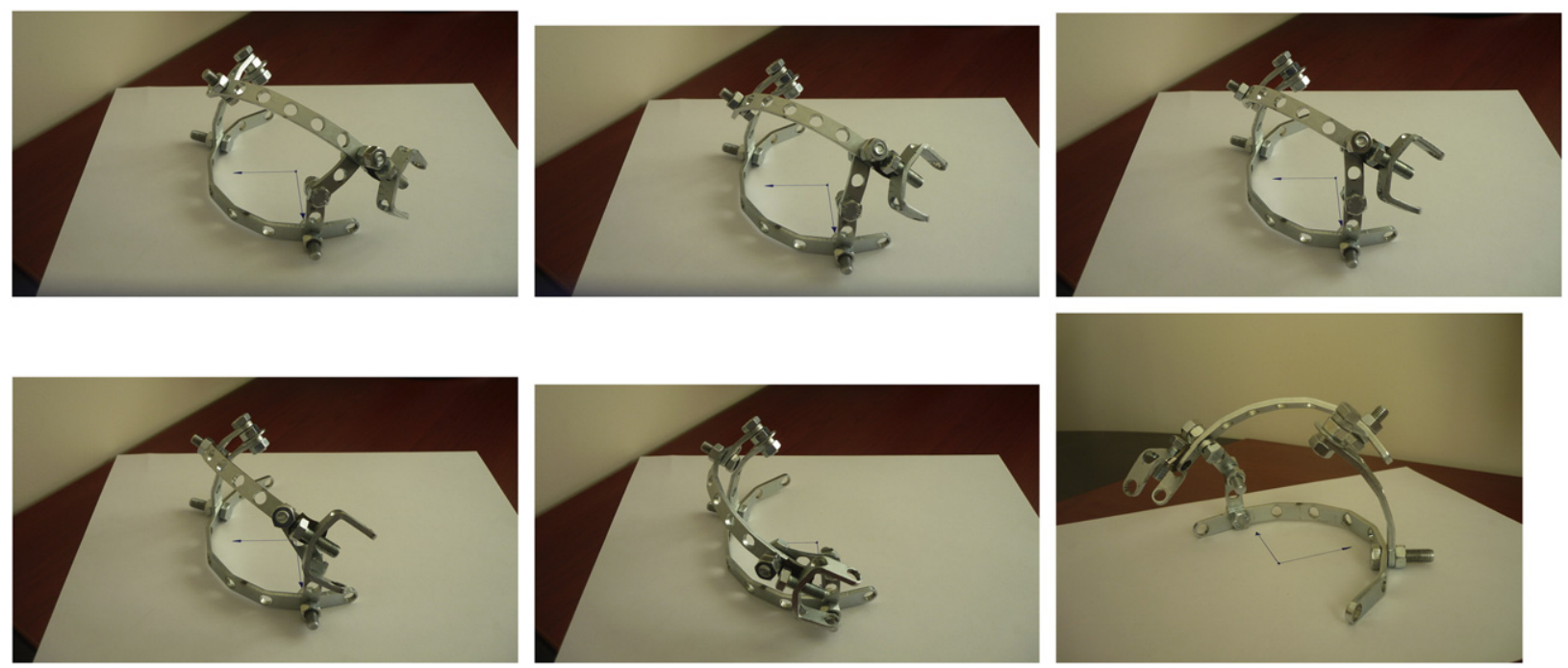

Fig. 7. The constructed spherical four-bar mechanism at different poses.

2. When we use the problem for $\mathrm{R}-\mathrm{R}$ open chain to construct a $4 \mathrm{R}$ spherical likage, we see that maximum four solutions can be obtained.

a. If we find only one real solution for R-R open chain, we cannot contruct $4 R$ spherical linkage.

b. If two real and physically realizable solutions are obtained, only one 4R spherical linkage can be constructed.

c. If three real and physically realizable solutions are calculated, then we can construct three different $4 \mathrm{R}$ spherical linkage that fulfills given motion.

d. If four real and physically realizable solutions are obtained, then maximum six different $4 \mathrm{R}$ spherical linkage can be constructed for required motion.

This is good to obtain several $4 \mathrm{R}$ spherical linkage for the same spherical motion.

3. When $4 \mathrm{R}$ mechanism is physically constructed after calculating design parameters, we observed that dividing $4 \mathrm{R}$ spherical linkage into two RR open chains make the assembly process easier. The design parameters are easily measured and therefore assembly process is more comfortable.

\section{References}

[1] N.I. Levitskii, Synthesis of Mechanisms by Chebyshev, USSR Academy of Science, 1946.

[2] J.R. Zimmerman, Four-precision synthesis of the spherical four-bar function generator, Mechanism and Machine Theory 2 (1967) 133-139.

[3] C.H. Chiang, Kinematic of Spherical Mechanisms, Krieger Publishing Company, Malabar, Florida, 2000.

[4] R. Alizade, Ö. Kilit, Analytical synthesis of function generating spherical four-bar mechanism for the five precision points, Mechanism and Machine Theory 40 (2005) 863-878.

[5] R. Alizade, Erkin Gezgin, Synthesis of function generating spherical four bar mechanism for the six independent parameters, Mechanism and Machine Theory 46 (2011) 1316-1326.

[6] J. Chu, J. Sun, Numerical atlas method for path generation of spherical four-bar mechanism, Mechanism and Machine Theory 45 (2010) 867-879.

[7] G. Mullineux, Atlas of spherical four-bar mechanism, Mechanism and Machine Theory 46 (2011) 1811-1823.

[8] O. Bottema, B. Roth, Theoretical Kinematics, North-Holland Publ., Amsterdam, Reprinted (1990), Dover, New York, 1979.

[9] R. Bodduluri, J.M. McCarthy, Finite position synthesis using the image curve of a spherical four-bar motion, ASME Journal of Mechanical Design 114 (1) (1992) 55-60.

[10] B. Ravani, B. Roth, Motion synthesis using kinematic mappings, ASME Journal of Mechanisms Transmissions and Automation in Design 105 (1) (1983) 460-467.

[11] J.M. McCarthy, R.M. Bodduluri, Avoiding singular configurations in finite position synthesis of spherical $4 \mathrm{R}$ linkages, Mechanism and Machine Theory 35 (2000) 451-462.

[12] D.M. Tse, P.M. Larochelle, Approximating spatial locations with spherical orientations for spherical mechanism design, ASME Journal of Mechanical Design 122 (4) (2000) 457-463.

[13] P.M. Larochelle, J. Dooley, P. Murray, J.M. McCarthy, SPHINX: software for synthesizing spherical 4R mechanisms, in: Proceeding of NSFD and MSC, Univ.of North Carolina at Charlotte, NC, 1, 1993, pp. 607-611.

[14] P.M. Larochelle, Approximate motion synthesis of open and closed chains via parametric constraint manifold fitting: preliminary results, in: Proceedings of DETC'03, ASME 2003, DETC-CIEC, Chicago, IL, USA, Sept. 2-6, 2003, pp. 1-9.

[15] K. Al-Widyan, J. Angeles, A robust solution to the spherical rigid body guidance problem, in: Proceedings of DETC'03, ASME 2003, DETC-CIEC, Chicago, IL, USA, Sept. 2-6, 2003, pp. 1-8.

[16] D.A. Ruth, J.M. McCarthy, The design of spherical 4R linkages for four specified orientations, Mechanism and Machine Theory 34 (1999) 677-692.

[17] K.H. Shirazi, Computer modeling and geometric construction for four point synthesis of 4R spherical linkages, Applied Mathematical Modelling 31 (2007) 1874-1888.

[18] W.-T. Lee, K. Russell, Q. Shen, R.S. Sodhi, On adjustable spherical four-bar motion generation for expanded prescribed positions, Mechanism and Machine Theory 44 (2009) 247-254. 